\title{
Glassy Effects for General Images
}

\author{
Gwanggil Jeon and Seokhoon Kang \\ Department of Embedded Systems Engineering, Incheon National University \\ 119 Academy-ro, Yeonsu-gu, Incheon 406-772, Korea \\ \{gjeon,hana\}@incheon.ac.kr
}

\begin{abstract}
This paper proposes a new grassy effect generation method. The grassy effect changes original image as the one behind the grass. Random number generation function is used to decide block size and the pixel location. Various block size and pixel location give unexpected grassy effect to the original image. Objective and visual performance comparison reveals that the proposed method successfully generates glassy effected images.
\end{abstract}

Keywords: Image processing, glassy effect, random number generation, variable length block

\section{Introduction}

With the fast computing devices, embedded systems and image processors available in the 21 st century, digital image processing field has achieved many missions [1,2]. Image processing yields an effect on the original image and perform to obtain desired image [35]. One of image processing effects is glassy effect (GE). The GE effect helps original image to look of glassy image.

The glass is an amorphous solid object which shows a glass-liquid transition. This is the reversible transition in amorphous objects from a rigid and fragile state into a melted or elastomer state. Therefore, glasses are generally fragile and are optically transparent. The GE is a use of computer methods to conduct image processing on digital signals. As a subcategory of digital image processing, it changes original image as the one behind the grass. Normally the GE is performed over 2D signal, however this can be modeled in the multi-dimensional signal [6-15].

Figure 1 shows the process of GE effect. The observer after GE effect may see the original image behind the glass. As one can see the GE effect applied image loses high frequency energy and the image look blurred.

In this paper, we propose a real time GE method. The rest of the paper is organized as follows. Section 2 describes the proposed GE method. Section 3 shows simulation results and discussion is provided. Finally, conclusion remarks are drawn in Section 4. 


\section{Proposed Method}

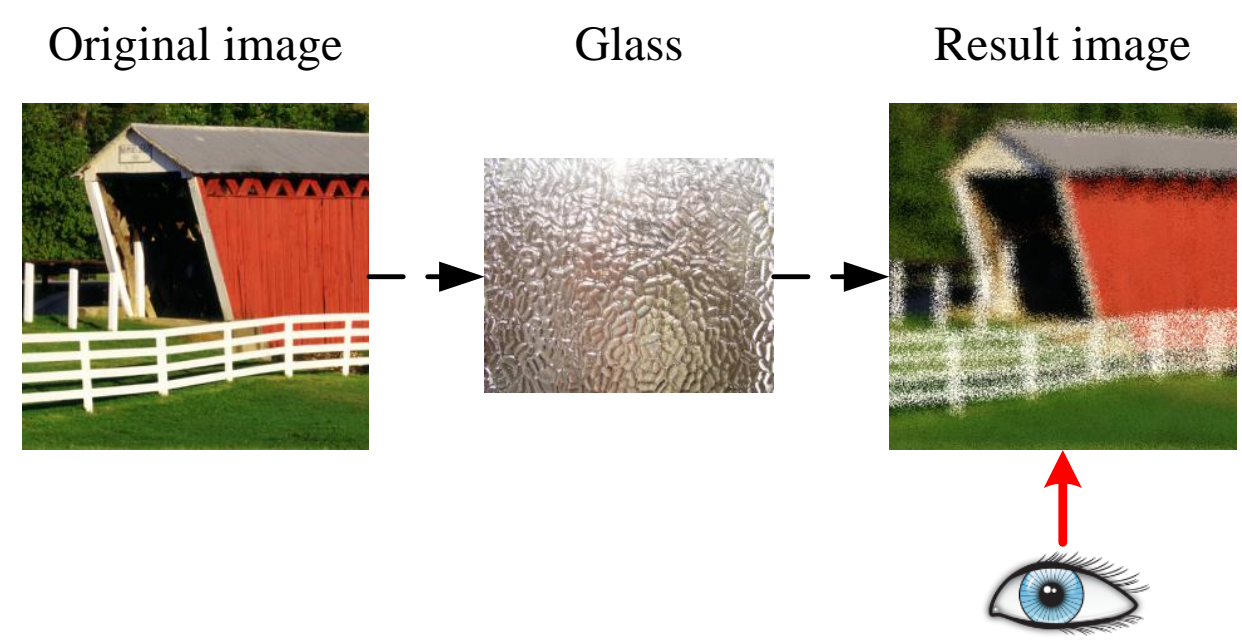

Figure 1. The Process of the Glassy Effect

The proposed method consists of three steps as shown in Figure 2. Here is pseudo code of the proposed method.

\section{Pseudo Code:}

\section{Step 1}

Generate two random numbers (both numbers $\leq 20$ ) and choose them as a horizontal and vertical block size $\left(b_{1}, b_{2}\right)$ for every pixel location. Parameters $c_{H}$ and $c_{V}$ are currently processed pixel position

$\mathrm{b} 1=\operatorname{ceil}(\operatorname{rand}(1) * 20)$;

b2 $=\operatorname{ceil}(\operatorname{rand}(1) * 20)$;

block = image $(\mathrm{cH}: \mathrm{cH}+\mathrm{b} 1-1, \mathrm{cv}: \mathrm{cv}+\mathrm{b} 2-1,:)$;

Step 2

Generate two random numbers and choose them as a horizontal and vertical pixel positions $\left(\mathrm{s}_{1}, \mathrm{~s}_{2}\right)$

$\mathrm{s} 1=\operatorname{ceil}(\operatorname{rand}(1) * \mathrm{~b} 1)$

$\mathrm{s} 2=\operatorname{ceil}(\operatorname{rand}(1) * \mathrm{~b} 2)$

Step 3

Select the corresponding pixel as a result one

Red_intensity $=$ image $(\mathrm{s} 1, \mathrm{~s} 1,1)$;

Green_intensity $=$ image $(\mathrm{s} 1, \mathrm{~s} 1,2)$;

Blue_intensity = image $(\mathrm{s} 1, \mathrm{~s} 1,3)$;

Step 4

The process is conducted for all pixels 
Input image

$\boldsymbol{\gamma}$

From 1 to end in vertical direction

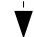

From $\mathrm{c}_{\mathrm{V}}=1$ to $\mathrm{c}_{\mathrm{V}}=\mathrm{end}$ in vertical direction

$\boldsymbol{7}$

From $\mathrm{c}_{\mathrm{H}}=1$ to $\mathrm{c}_{\mathrm{H}}=$ end in horizontal direction

Step 2: generate two random numbers for $\left(\mathrm{s}_{1}, \mathrm{~s}_{2}\right)$

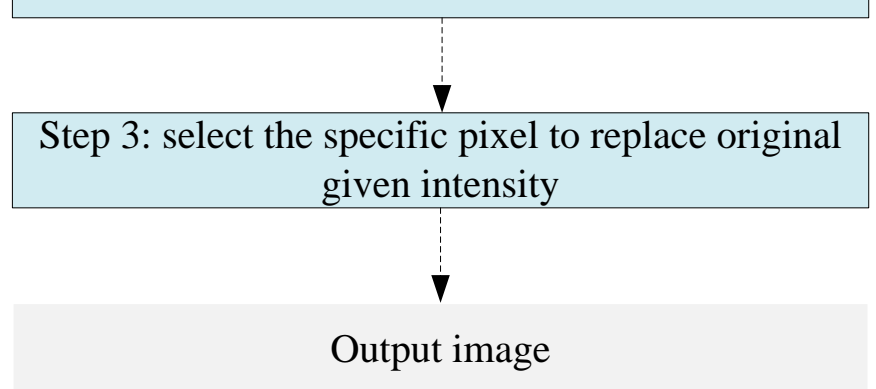

Figure 2. Block Diagram of the Proposed Method

Let us assume $18^{\text {th }}$ McM image as the test image and the pixel location $\left(c_{H}, c_{V}\right)=(493$, 493). After applying Step 1, one obtains $\left(b_{1}, b_{2}\right)=(7,7)$. The results blocks for red, green, and blue are shown as Figure 3.

\begin{tabular}{rrrrrrr}
\hline 94 & 95 & 80 & 75 & 80 & 92 & 90 \\
105 & 114 & 98 & 96 & 103 & 105 & 106 \\
114 & 115 & 116 & 105 & 102 & 96 & 101 \\
126 & 118 & 115 & 120 & 109 & 106 & 95 \\
119 & 124 & 115 & 109 & 108 & 115 & 109 \\
101 & 105 & 112 & 92 & 106 & 120 & 114 \\
87 & 77 & 89 & 92 & 105 & 119 & 122
\end{tabular}

$\begin{array}{lllllll}123 & 123 & 118 & 105 & 104 & 120 & 122 \\ 128 & 137 & 129 & 123 & 129 & 133 & 133 \\ 131 & 138 & 136 & 128 & 131 & 132 & 130 \\ 138 & 138 & 136 & 139 & 138 & 138 & 126 \\ 132 & 145 & 139 & 134 & 137 & 142 & 129 \\ 122 & 131 & 137 & 118 & 131 & 143 & 129 \\ 111 & 102 & 117 & 121 & 129 & 139 & 137\end{array}$

(a)

\begin{tabular}{lllllll}
\hline 41 & 42 & 34 & 29 & 29 & 43 & 44 \\
44 & 50 & 46 & 38 & 40 & 45 & 50 \\
47 & 45 & 47 & 41 & 42 & 43 & 45 \\
51 & 44 & 45 & 47 & 49 & 48 & 39 \\
50 & 53 & 46 & 42 & 48 & 51 & 43 \\
41 & 45 & 45 & 38 & 44 & 48 & 45 \\
34 & 30 & 39 & 40 & 40 & 45 & 51
\end{tabular}

(b)

Figure 3. (a) Red Channel, (b) Green Channel, and (c) Blue Channel of Selected Block 
After Step 2, one may obtain $\left(\mathrm{s}_{1}, \mathrm{~s}_{2}\right)$ which is pixel location in a block. Note that parameters $s_{1}$ and $s_{2}$ are smaller than $b_{1}$ and $b_{2}$, respectively. When $\left(s_{1}, s_{2}\right)=(4,4)$, color components are obtained as follows:

Red_intensity = image $(497,497,1)$;

Green_intensity = image $(497,497,2)$;

Blue_intensity = image $(497,497,3)$;

As $(\mathrm{cH}, \mathrm{cV})=(493,493)$, pixel $(497,497)$ is located 4 pixels to the right and 4 pixels to the down, respectively. Now, the red, green, and blue intensities at $(497,497)$ position is $(120,139,47)$. This process is shown in Figure 4, which is the results of Step 3.

$\begin{array}{rrrrrrr}94 & 95 & 80 & 75 & 80 & 92 & 90 \\ 105 & 114 & 98 & 96 & 103 & 105 & 106 \\ 114 & 115 & 116 & 105 & 102 & 96 & 101 \\ 126 & 118 & 115 & 120 & 109 & 106 & 95 \\ 119 & 124 & 115 & 109 & 108 & 115 & 109 \\ 101 & 105 & 112 & 92 & 106 & 120 & 114 \\ 87 & 77 & 89 & 92 & 105 & 119 & 122\end{array}$

(a)

$\begin{array}{lllllll}123 & 123 & 118 & 105 & 104 & 120 & 122 \\ 128 & 137 & 129 & 123 & 129 & 133 & 133 \\ 131 & 138 & 136 & 128 & 131 & 132 & 130 \\ 138 & 138 & 136 & 139 & 138 & 138 & 126 \\ 132 & 145 & 139 & 134 & 137 & 142 & 129 \\ 122 & 131 & 137 & 118 & 131 & 143 & 129 \\ 111 & 102 & 117 & 121 & 129 & 139 & 137\end{array}$

(b)

$\begin{array}{lllllll}41 & 42 & 34 & 29 & 29 & 43 & 44 \\ 44 & 50 & 46 & 38 & 40 & 45 & 50 \\ 47 & 45 & 47 & 41 & 42 & 43 & 45 \\ 51 & 44 & 45 & 47 & 49 & 48 & 39 \\ 50 & 53 & 46 & 42 & 48 & 51 & 43 \\ 41 & 45 & 45 & 38 & 44 & 48 & 45 \\ 34 & 30 & 39 & 40 & 40 & 45 & 51\end{array}$

(c)

Figure 4. Selected Red, Green, and Blue Channel Intensity

Finally, the original pixel $(94,123,41)$ is replaced by $(120,139,47)$ for performing GE.

\section{Simulation Results}

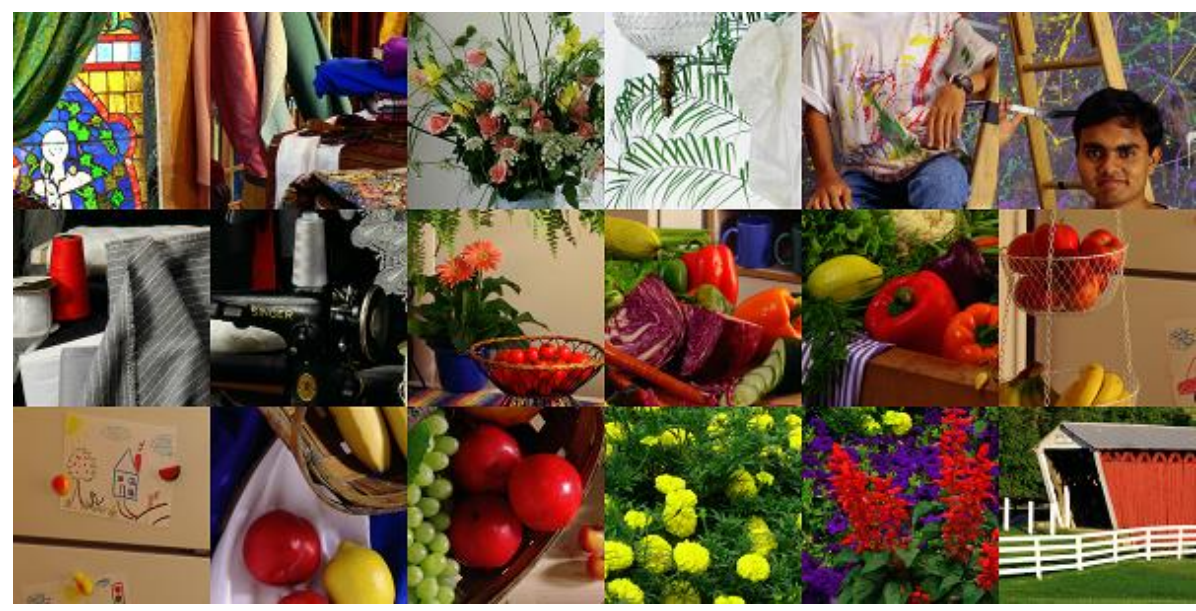

Figure 5. McM Dataset

We experiment with $18 \mathrm{McM}$ images, which is shown in Figure 5. Tables 1-3 show the mean squared error (MSE) and the peak signal-to-noise ratio (PSNR) performance results for different $\left(s_{1}, s_{2}\right)$ combinations. Note that $\left(s_{1}, s_{2}\right)$ are computed with random number generating function. However to compare the performance, we selected three 
conditions of $\left(s_{1}, s_{2}\right)$ parameters. Each table has four MSE and four PSNR results which are for red, green, blue, and color information, respectively.

Tables 1-3 are results with $\left(\mathrm{s}_{1}, \mathrm{~s}_{2}\right)=(5,5),\left(\mathrm{s}_{1}, \mathrm{~s}_{2}\right)=(10,10)$, and $\left(\mathrm{s}_{1}, \mathrm{~s}_{2}\right)=(20,20)$, respectively.

Table 1. MSE and PSNR Results of $\left(s_{1}, s_{2}\right)=(5,5)$

\begin{tabular}{|c|c|c|c|c|c|c|c|c|}
\hline & \multicolumn{5}{|c}{ MSE } & \multicolumn{4}{c|}{ PSNR } \\
\hline & Red & Green & Blue & Color & Red & Green & Blue & Color \\
\hline 1 & 4085.29 & 4926.19 & 4965.01 & 4658.83 & 12.019 & 11.206 & 11.172 & 11.448 \\
\hline 2 & 2814.61 & 2688.54 & 2301.28 & 2601.48 & 13.637 & 13.836 & 14.511 & 13.979 \\
\hline 3 & 3580.38 & 2882.91 & 3699.52 & 3387.60 & 12.592 & 13.532 & 12.449 & 12.832 \\
\hline 4 & 5492.03 & 2973.79 & 6039.08 & 4834.96 & 10.733 & 13.398 & 10.321 & 11.287 \\
\hline 5 & 1518.36 & 2169.48 & 2762.50 & 2150.11 & 16.317 & 14.767 & 13.718 & 14.806 \\
\hline 6 & 1591.63 & 1438.49 & 1234.70 & 1421.61 & 16.112 & 16.552 & 17.215 & 16.603 \\
\hline 7 & 2161.87 & 2128.85 & 2035.76 & 2108.82 & 14.783 & 14.849 & 15.044 & 14.890 \\
\hline 8 & 2031.17 & 1671.25 & 1397.86 & 1700.09 & 15.053 & 15.900 & 16.676 & 15.826 \\
\hline 9 & 2346.30 & 1377.16 & 1178.33 & 1633.93 & 14.427 & 16.741 & 17.418 & 15.998 \\
\hline 10 & 1653.60 & 1525.86 & 1188.29 & 1455.92 & 15.946 & 16.296 & 17.382 & 16.499 \\
\hline 11 & 1681.07 & 1042.14 & 495.24 & 1072.82 & 15.875 & 17.952 & 21.183 & 17.826 \\
\hline 12 & 1066.49 & 1476.94 & 909.69 & 1151.04 & 17.851 & 16.437 & 18.542 & 17.520 \\
\hline 13 & 742.62 & 728.81 & 465.09 & 645.51 & 19.423 & 19.505 & 21.455 & 20.032 \\
\hline 14 & 928.76 & 872.26 & 961.96 & 921.00 & 18.452 & 18.724 & 18.299 & 18.488 \\
\hline 15 & 1282.14 & 949.09 & 748.12 & 993.11 & 17.051 & 18.358 & 19.391 & 18.161 \\
\hline 16 & 3272.25 & 3346.86 & 461.54 & 2360.22 & 12.982 & 12.884 & 21.489 & 14.401 \\
\hline 17 & 3440.21 & 1844.32 & 1710.74 & 2331.76 & 12.765 & 15.472 & 15.799 & 14.454 \\
\hline 18 & 6014.00 & 5709.69 & 7077.96 & 6267.22 & 10.339 & 10.565 & 9.632 & 10.160 \\
\hline Avg. & 2539.04 & 2208.48 & 2201.81 & 2316.45 & 14.80 & 15.39 & 16.21 & 15.29 \\
\hline
\end{tabular}

Table 2. MSE and PSNR Results of $\left(s_{1}, s_{2}\right)=(10,10)$

\begin{tabular}{|c|c|c|c|c|c|c|c|c|}
\hline & \multicolumn{5}{|c|}{ MSE } & \multicolumn{4}{c|}{ PSNR } \\
\hline & Red & Green & Blue & Color & Red & Green & Blue & Color \\
\hline 1 & 3185.82 & 3902.09 & 3801.63 & 3629.85 & 13.099 & 12.218 & 12.331 & 12.532 \\
\hline 2 & 2062.37 & 1965.39 & 1701.77 & 1909.85 & 14.987 & 15.196 & 15.822 & 15.321 \\
\hline 3 & 3019.46 & 2465.73 & 3177.35 & 2887.51 & 13.332 & 14.211 & 13.110 & 13.526 \\
\hline 4 & 4726.22 & 2545.87 & 5188.34 & 4153.47 & 11.386 & 14.072 & 10.981 & 11.947 \\
\hline 5 & 1145.15 & 1700.02 & 2211.91 & 1685.69 & 17.542 & 15.826 & 14.683 & 15.863 \\
\hline 6 & 1191.31 & 1112.81 & 964.23 & 1089.45 & 17.371 & 17.667 & 18.289 & 17.759 \\
\hline
\end{tabular}




\begin{tabular}{|c|c|c|c|c|c|c|c|c|}
\hline 7 & 1561.20 & 1545.81 & 1492.71 & 1533.24 & 16.196 & 16.239 & 16.391 & 16.275 \\
\hline 8 & 1565.12 & 1303.23 & 1083.35 & 1317.23 & 16.185 & 16.981 & 17.783 & 16.934 \\
\hline 9 & 1872.41 & 1089.81 & 964.65 & 1308.96 & 15.407 & 17.757 & 18.287 & 16.962 \\
\hline 10 & 1242.09 & 1134.46 & 896.65 & 1091.07 & 17.189 & 17.583 & 18.605 & 17.752 \\
\hline 11 & 1232.79 & 825.60 & 377.59 & 811.99 & 17.222 & 18.963 & 22.361 & 19.035 \\
\hline 12 & 804.97 & 1171.83 & 745.05 & 907.28 & 19.073 & 17.442 & 19.409 & 18.553 \\
\hline 13 & 568.01 & 550.07 & 360.38 & 492.82 & 20.587 & 20.727 & 22.563 & 21.204 \\
\hline 14 & 699.98 & 646.30 & 693.07 & 679.78 & 19.680 & 20.026 & 19.723 & 19.807 \\
\hline 15 & 932.07 & 678.51 & 531.46 & 714.01 & 18.436 & 19.815 & 20.876 & 19.594 \\
\hline 16 & 2582.12 & 2727.27 & 410.16 & 1906.52 & 14.011 & 13.774 & 22.001 & 15.328 \\
\hline 17 & 2688.14 & 1475.01 & 1373.61 & 1845.58 & 13.836 & 16.443 & 16.752 & 15.469 \\
\hline 18 & 4273.64 & 4065.75 & 4898.84 & 4412.74 & 11.823 & 12.039 & 11.230 & 11.684 \\
\hline Avg. & 1964.05 & 1716.98 & 1715.15 & 1798.73 & 15.96 & 16.50 & 17.29 & 16.42 \\
\hline
\end{tabular}

Table 3. MSE and PSNR Results of $\left(s_{1}, s_{2}\right)=(20,20)$

\begin{tabular}{|c|c|c|c|c|c|c|c|c|}
\hline & \multicolumn{6}{|c|}{ MSE } & \multicolumn{4}{c|}{ PSNR } \\
\hline & Red & Green & Blue & Color & Red & Green & Blue & Color \\
\hline 1 & 2980.42 & 3664.85 & 3573.78 & 3406.35 & 13.388 & 12.490 & 12.600 & 12.808 \\
\hline 2 & 1921.09 & 1820.09 & 1595.19 & 1778.79 & 15.295 & 15.530 & 16.103 & 15.630 \\
\hline 3 & 2915.86 & 2398.89 & 3111.60 & 2808.78 & 13.483 & 14.331 & 13.201 & 13.646 \\
\hline 4 & 4640.98 & 2503.73 & 5135.94 & 4093.55 & 11.465 & 14.145 & 11.025 & 12.010 \\
\hline 5 & 1088.29 & 1539.11 & 2033.13 & 1553.51 & 17.763 & 16.258 & 15.049 & 16.218 \\
\hline 6 & 1123.35 & 1050.03 & 905.24 & 1026.21 & 17.626 & 17.919 & 18.563 & 18.018 \\
\hline 7 & 1347.31 & 1342.86 & 1298.90 & 1329.69 & 16.836 & 16.851 & 16.995 & 16.893 \\
\hline 8 & 1476.69 & 1230.33 & 1022.80 & 1243.27 & 16.438 & 17.231 & 18.033 & 17.185 \\
\hline 9 & 1778.23 & 1044.42 & 954.78 & 1259.15 & 15.631 & 17.942 & 18.332 & 17.130 \\
\hline 10 & 1207.16 & 1025.43 & 827.76 & 1020.12 & 17.313 & 18.022 & 18.952 & 18.044 \\
\hline 11 & 1067.24 & 716.23 & 318.16 & 700.54 & 17.848 & 19.580 & 23.104 & 19.676 \\
\hline 12 & 744.12 & 1079.28 & 691.80 & 838.40 & 19.414 & 17.799 & 19.731 & 18.896 \\
\hline 13 & 548.66 & 533.10 & 349.95 & 477.24 & 20.738 & 20.863 & 22.691 & 21.343 \\
\hline 14 & 765.29 & 682.32 & 663.08 & 703.56 & 19.293 & 19.791 & 19.915 & 19.658 \\
\hline 15 & 860.24 & 612.74 & 482.06 & 651.68 & 18.785 & 20.258 & 21.300 & 19.990 \\
\hline 16 & 2480.33 & 2638.00 & 397.75 & 1838.69 & 14.186 & 13.918 & 22.135 & 15.486 \\
\hline 17 & 2511.39 & 1374.44 & 1278.33 & 1721.39 & 14.132 & 16.750 & 17.064 & 15.772 \\
\hline 18 & 3894.69 & 3690.17 & 4328.51 & 3971.12 & 12.226 & 12.460 & 11.767 & 12.142 \\
\hline
\end{tabular}



6.

\begin{tabular}{|l|l|l|l|l|l|l|l|l|}
\hline Avg. & 1852.85 & 1608.11 & 1609.38 & 1690.11 & 16.21 & 16.79 & 17.59 & 16.70 \\
\hline
\end{tabular}

The MSE and PSNR results of color information of Tables 1-3 can be drawn as Figure

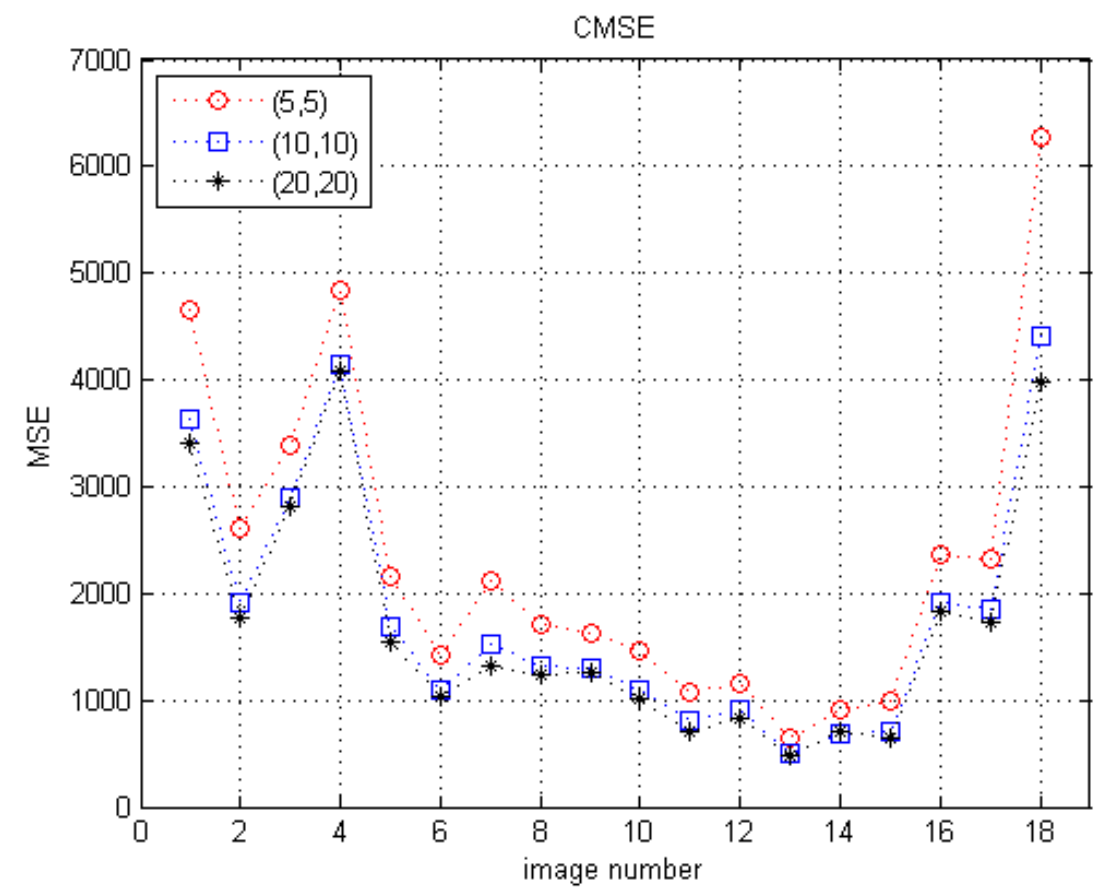

(a)

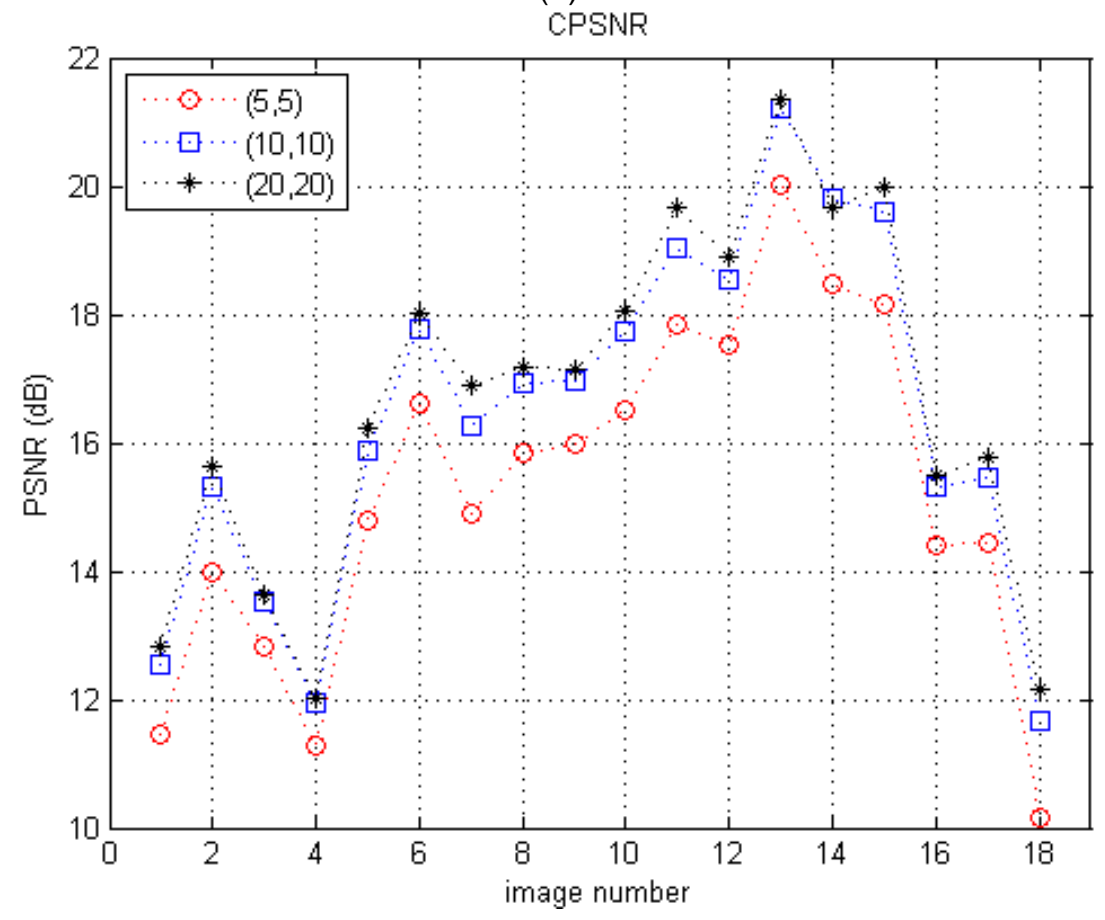

(b)

Figure 6. Performance Comparison on Three $\left(s_{1}, s_{2}\right)$ Conditions: (a) CMSE, (b) CPSNR 
The visual performance is compared in Figures. 7-10. As we can see, image with higher $\left(\mathrm{s}_{1}, \mathrm{~s}_{2}\right)$ are more blur, and the high frequency energy was lost.

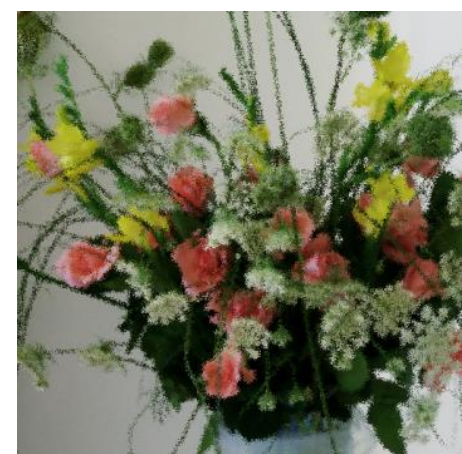

(a)

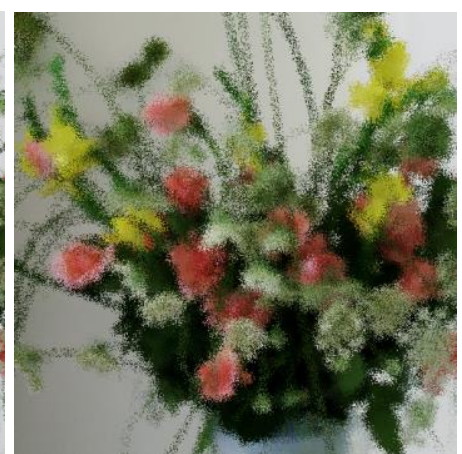

(b)

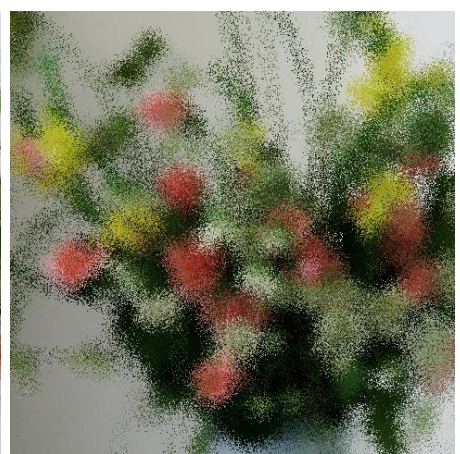

(c)

Figure 7. Visual Performance Comparison for \#3 McM Image: (a) $\left(s_{1}, s_{2}\right)=(5$, $5),(b)\left(s_{1}, s_{2}\right)=(10,10)$, and $(c)\left(s_{1}, s_{2}\right)=(20,20)$

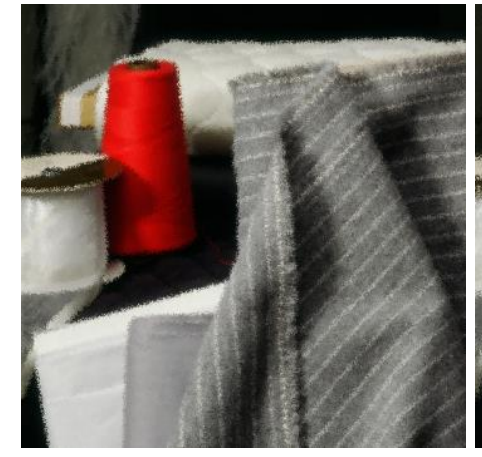

(a)

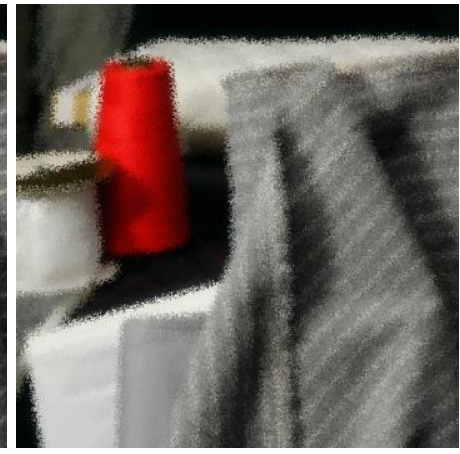

(b)

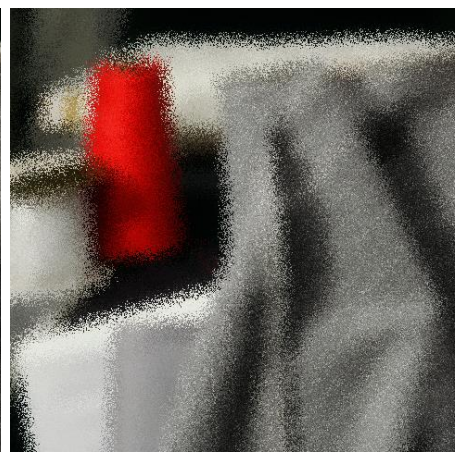

(c)

Figure 8. Visual Performance Comparison for \#7 McM Image: (a) $\left(s_{1}, s_{2}\right)=(5$, 5), (b) $\left(s_{1}, s_{2}\right)=(10,10)$, and $(c)\left(s_{1}, s_{2}\right)=(20,20)$

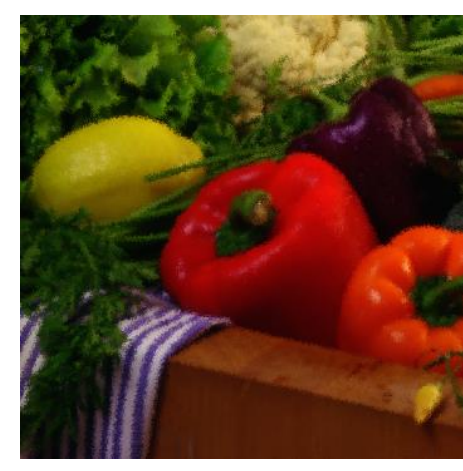

(a)

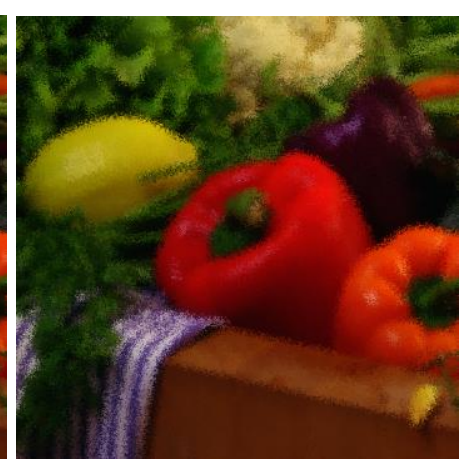

(b)

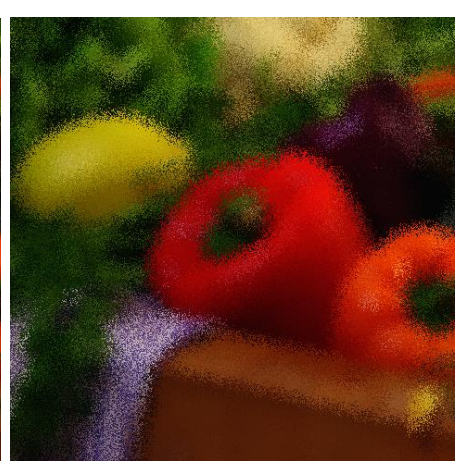

(c)

Figure 9. Visual Performance Comparison for \#11 McM Image: (a) $\left(s_{1}, s_{2}\right)=$ $(5,5),(b)\left(s_{1}, s_{2}\right)=(10,10)$, and $(c)\left(s_{1}, s_{2}\right)=(20,20)$ 


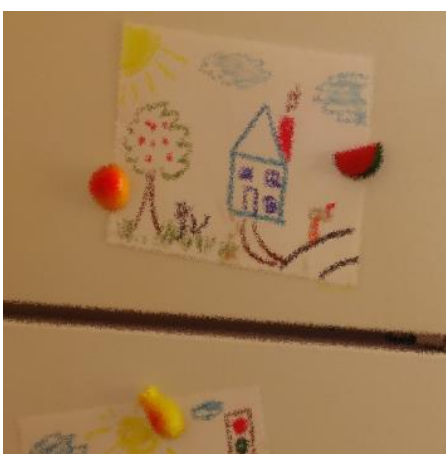

(a)

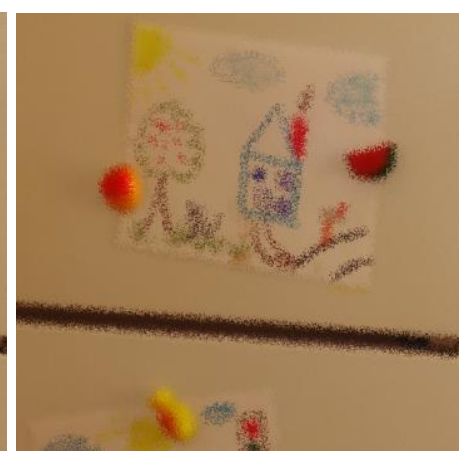

(b)

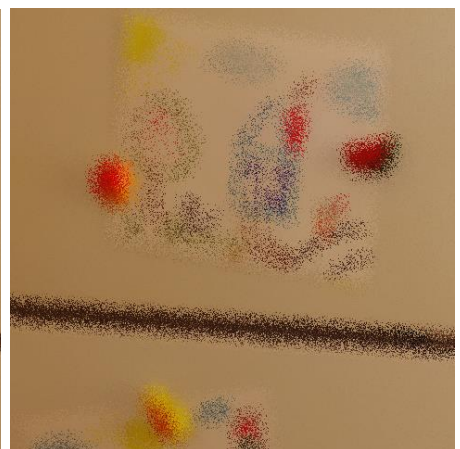

(c)

Figure 10. Visual Performance Comparison for \#13 McM Image: (a) $\left(s_{1}, s_{2}\right)=$ $(5,5),(b)\left(s_{1}, s_{2}\right)=(10,10)$, and $(c)\left(s_{1}, s_{2}\right)=(20,20)$

\section{Conclusions}

In this paper, we presented a new grassy effect method which make original image look blurred. Random number generator was used for block size and pixel location selection. Different block size and pixel location parameters provide various grassy effects. Simulation results reveals that the presented method successfully provide glassy effected images.

\section{Acknowledgements}

This work was supported by the Incheon National University Research Grant in 2012.

\section{Footnote}

This paper is a revised and expanded version of a paper entitled "Block-based Glassy Effects for Natural Images" presented at "The 6th International Conference on Multimedia, Computer Graphics and Broadcasting 2014."

\section{References}

[1] Kelsey J., Schneier B., Wagner D. and Hall C., "Cryptanalytic attacks on pseudorandom number generators," 5th Fast Software Encryption International Workshop, (1998).

[2] Rafael C. G. and Richard E. W., "Digital Image Processing," Third Edition, Prentice Hall, (2008).

[3] William K. P., "Digital Image Processing," Third Edition, Wiley, (2001).

[4] Li S., Zhang B. and Sun H., "Real-Time Restoration for Motion-Blurred Color Images by Multichannel Combination Processing," IEEE ETCS 2009, vol. 1, (2009).

[5] Kaulgud N. and Desai U. B., "Restoration of color images subjected to interchannel blurring," IEEE ISCAS '99, vol. 4, (1999).

[6] Lasang P., Chin O. and Sheng M. S., "CFA-based motion blur removal using long/short exposure pairs,” IEEE Trans. Consumer Electronics, vol. 56 no. 2, May (2010), pp. 332-338.

[7] R. Wang and Q. Lin, "The research of foggy blurring image restoration," IEEE ICCTA 2011, (2011), pp. 782-786.

[8] Cong J., Qing H. D. and Jun L., "Computer Virus Propagation Model Based on Variable Propagation Rate," IJAST, vol. 1, December (2008), pp. 29-34.

[9] Ching T. H., Yeh K. W. and Kuo M. H., "Hybrid Watermarking Scheme for Halftone Images," IJAST, vol. 1, December (2008), pp. 9-20.

[10] S. Lee, W. Yun and G. Jeon., "Glassy Effects for General Images," in Proc. MulGraB 2014, December (2014).

[11] G. Jeon, M. Anisetti, V. Bellandi and J. Jeong, "Fuzzy rule-based edge-restoration algorithm in HDTV interlaced sequences,” IEEE Trans. Consumer Electronics, vol. 53 no. 2, May (2007), pp. 725-731.

[12] G. Jeon, M. Anisetti, V. Bellandi, E. Damiani and J. Jeong, "Rough sets-assisted subfield optimization for alternating current plasma display panel," IEEE Trans. Consumer Electronics, vol. 53 no. 3, August (2007), pp. 825-832. 
[13] G. Jeon, M. Anisetti, V. Bellandi, E. Damiani and J. Jeong, "Fuzzy weighted approach to improve visual quality of edge-based filtering," IEEE Trans. Consumer Electronics, vol. 53 no. 4, (2007), pp. 1661-1667, November.

[14] G. Jeon, M. Anisetti, J. Lee, V. Bellandi, E. Damiani and J. Jeong, "Concept of linguistic variablebased fuzzy ensemble approach: application to interlaced HDTV sequences," IEEE Trans. Fuzzy Systems, vol. 17 no. 6, December (2009), pp. 1245-1258.

[15] G. Jeon, M. Y. Jung, M. Anisetti, V. Bellandi, E. Damiani and J. Jeong, "Specification of the geometric regularity model for fuzzy if-then rule based deinterlacing," IEEE/OSA Journal of Display Technology, vol. 6 no. 6, June (2010), pp. 235-243.

\section{Authors}

Gwanggil Jeon, received the BS, MS, and $\mathrm{PhD}$ (summa cum laude) degrees in Department of Electronics and Computer Engineering from Hanyang University, Seoul, Korea, in 2003, 2005, and 2008, respectively.

From 2008 to 2009, he was with the Department of Electronics and Computer Engineering, Hanyang University, from 2009 to 2011, he was with the School of Information Technology and Engineering (SITE), University of Ottawa, as a postdoctoral fellow, and from 2011 to 2012, he was with the Graduate School of Science \& Technology, Niigata University, as an assistant professor. He is currently an assistant professor with the Department of Embedded Systems Engineering, Incheon National University, Incheon, Korea. His research interests fall under the umbrella of image processing, particularly image compression, motion estimation, demosaicking, and image enhancement as well as computational intelligence such as fuzzy and rough sets theories.

He was the recipient of the IEEE Chester Sall Award in 2007 and the 2008 ETRI Journal Paper Award.

Seokhoon Kang, received the BS, MS, and PhD degrees in Department of Electronic Communication Engineering from Hanyang University, Seoul, Korea, in 1989, 1991, and 1995, respectively. From 1996 to 2003, he was with the Department of Electronics and Computer Engineering, Dongseo University, Pusan, Korea, as an associate professor. He is currently a professor with the Department of Embedded Systems Engineering, Incheon National University, Incheon, Korea. His research interests include real time system, image processing, and mobile system. 